\title{
Up to
}

National Cancer Institute

\section{Source}

National Cancer Institute. Up to. NCI Thesaurus. Code C64643.

Used to indicate a limit or boundary. 